- Attaining an accurate prognosis of periodontally involved teeth is problematic.

- The reporting of success rates in endodontic literature is confused by the definition of 'success/failure'.

- Heroic attempts to maintain teeth with poor prognosis should be questioned. Implants should not be 'the last resort' and only used when all conventional therapies have failed.

\title{
Factors that affect individual tooth prognosis and choices in contemporary treatment planning
}

\author{
N. Mordohai, ${ }^{1}$ M. Reshad ${ }^{2}$ S. Jivraj $^{3}$ and W. Chee $^{4}$
}

\begin{abstract}
A clinician evaluates a tooth for its quality of health. Once accomplished an estimate of longevity and survival is estimated. With current knowledge about the survival and success of implants a decision is made as to whether to extract or to treat and maintain the tooth. Questions and doubts abound in the decision making process in regards to the prognosis of an individual tooth. Unfortunately in dentistry, as in all biologic sciences, there are no straightforward answers to questions. This article will attempt to review the literature in this area to aid the practitioner in the decision making process with regards to the compromised tooth. The article will focus on the single tooth or implant restoration. Other factors such as the strategic value of a tooth and financial limitations in relation to long-term prognosis will also be discussed.
\end{abstract}

\begin{tabular}{l} 
IMPLANTS \\
\hline 1. Rationale for dental implants \\
\hline 2. Treatment planning of implants in \\
posterior quadrants \\
\hline 3. Treatment planning of implants in \\
the aesthetic zone \\
\hline $\begin{array}{l}\text { 4. Surgical guidelines for dental } \\
\text { implant placement }\end{array}$ \\
\hline 5. Immediate implant placement: \\
treatment planning and surgical steps \\
for successful outcomes \\
\hline 6. Treatment planning of the \\
edentulous maxilla \\
\hline 7. Treatment planning of the \\
edentulous mandible \\
\hline 8. Impressions techniques for implant \\
dentistry \\
\hline 9. Screw versus cemented implant \\
supported restorations \\
\hline 10. Designing abutments for \\
cement retained implant supported \\
restorations \\
\hline 11. Connecting implants to teeth \\
\hline 12. Transitioning a patient from teeth \\
to implants \\
\hline 13. The role of orthodontics in implant \\
dentistry \\
\hline 14. Interdisciplinary approach to \\
implant dentistry \\
15. Factors that affect individual \\
tooth prognosis and choices in \\
contemporary treatment planning \\
16. Maintenance and failures \\
\hline
\end{tabular}

\section{INTRODUCTION}

Periodontally involved teeth receive multiple therapeutic procedures to arrest the disease, prevent attachment loss and possibly gain some attachment. Non-surgical and surgical endodontic therapy is performed on teeth with necrotic pulps to seal the tooth or re-seal the 'already sealed' root canal. On occasion a given tooth may require both periodontal and endodontic procedures followed by restoration to form and function. Today implant dentistry has shifted the treatment planning paradigm; questionable teeth may be extracted more frequently in favour of implant placement. 'Herodontics' is discouraged when the prognosis is poor or failure of treatment may result in inadequate bone for implant placement. Considerable thought

\footnotetext{
1,"2Resident Advanced Prosthodontics, USC School of Dentistry, 925 West $34^{\text {th }}$ St, Rm 116, Los Angeles, CA 90089, USA; ${ }^{3}$ Chairman, Section of Fixed Prosthodontics and Operative Dentistry, University of Southern California School of Dentistry / Private Prosthodontics Practitioner, Burbank, California, USA; ${ }^{4}$ Ralph W. and Jean L. Bleak Professor of Restorative Dentistry, Director of Implant Dentistry at the University of Southern California School of Dentistry / Private Prosthodontics Practitioner, Pasadena, California, USA ${ }^{*}$ Correspondence to: Dr Nikitas Mordohai, 73 Ermou Street, Thessaloniki, 54623, Greece

Email:nmordohai@hotmail.com
}

\section{Refereed Paper}

( $)$ British Dental Journal 2007; 202: 63-72

DOI: $10.1038 /$ bdj.2007.23 has to be given to prognosis from both a periodontal and an endodontic perspective. Therapeutic decisions need to be made based on this prognosis so that success in the long term can be achieved.

\section{Prognosis of periodontally involved teeth}

Attaining an accurate prognosis of periodontally involved teeth is problematic. Hirschfeld and Wasserman re-examined over 15,000 teeth in 600 patients with advanced periodontitis, at least 15 years after receiving treatment. The patients were generally well motivated in their personal and professional dental care. They also had similar periodontal involvement at the onset and received the same treatment. However, the patients differed markedly in post-treatment course, with tooth loss ranging from zero to 23 teeth per patient. ${ }^{1}$ In other words, it is almost impossible to predict the chance of survival of a periodontally compromised tooth.

In an attempt to establish clinical parameters that would lead to consistently correct prognoses, McGuire and Nunn published a series of papers. All articles were based on 100 patients with 2,509 teeth under maintenance care for up to 15 years. It became obvious that 'projections relying on the commonly taught clinical parameters were ineffective in predicting any outcome other than good'.2 Although the regression model formulated predicted accurately $81 \%$ of the time, its accuracy dropped to approximately 


\section{PRACTICE}

Fig. 1 Status before periodontal treatment reveals mild to moderate involvement of the incisors.

Fig. 2 Three-year post-operatively there is evidence of advanced periodontal destruction.
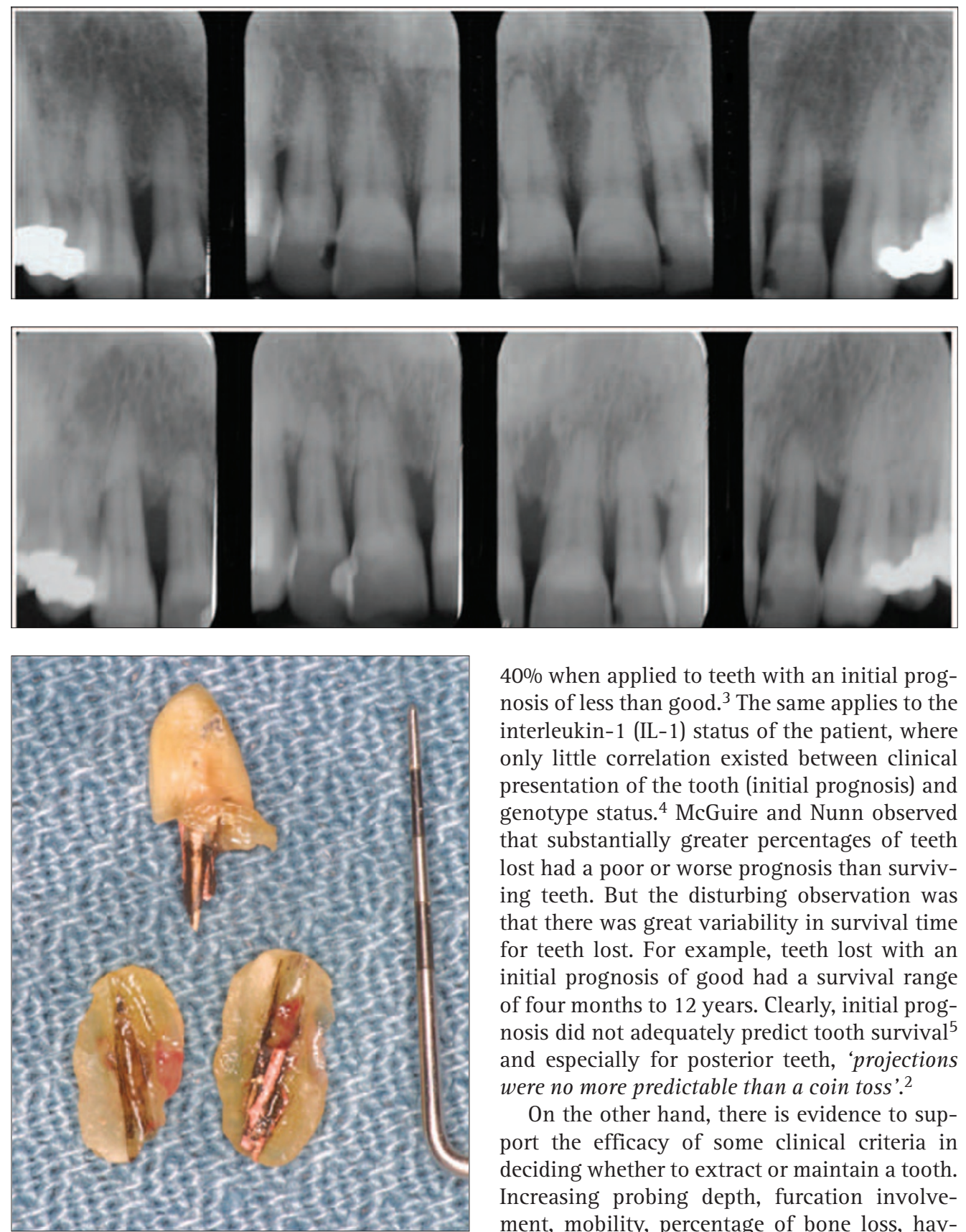

Fig. 3 Horizontal and vertical root fractures of mandibular lateral incisor that was endodontically treated and restored with a cast dowel.

Fig. 4 Maxillary first bicuspids are also prone to vertical fractures especially if restored with widediameter dowels.

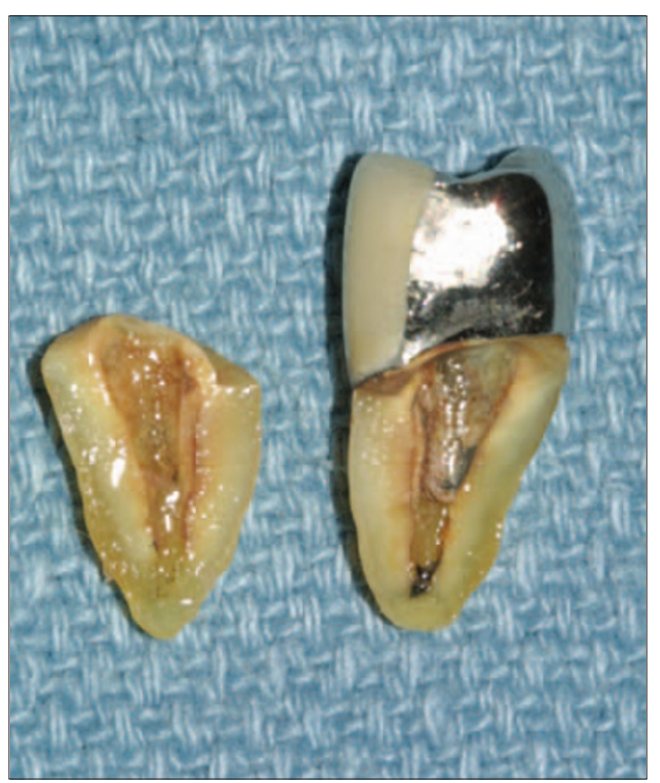

40\% when applied to teeth with an initial prognosis of less than good. ${ }^{3}$ The same applies to the interleukin-1 (IL-1) status of the patient, where only little correlation existed between clinical presentation of the tooth (initial prognosis) and genotype status. ${ }^{4}$ McGuire and Nunn observed that substantially greater percentages of teeth lost had a poor or worse prognosis than surviving teeth. But the disturbing observation was that there was great variability in survival time for teeth lost. For example, teeth lost with an initial prognosis of good had a survival range of four months to 12 years. Clearly, initial prognosis did not adequately predict tooth survival ${ }^{5}$ and especially for posterior teeth, "projections were no more predictable than a coin toss. ${ }^{2}$

On the other hand, there is evidence to support the efficacy of some clinical criteria in deciding whether to extract or maintain a tooth. Increasing probing depth, furcation involvement, mobility, percentage of bone loss, having a parafunctional habit and not wearing an occlusal splint and smoking resulted in an increased risk of tooth loss. ${ }^{5}$ Lang et al. found a highly significant relationship between increasing probing depth and increasing bleeding on probing (BOP) incidence and also a highly significant relationship between an increasing BOP and loss of probing attachment. Specifically, absence of BOP showed an almost 0\% risk for periodontal breakdown, while pockets that constantly bled during follow-up appointments had a 30\% risk for losing probing attachment. Although this number is still low, BOP still represents the most reliable clinical predictor for disease 'activity' during periodontal maintenance. ${ }^{6}$ Wasserman et al. confirmed the limited importance of BOP. Patients with periodontal breakdown had gingival inflammation more often than patients without breakdown. However, the teeth with the most inflammation did not necessarily correspond with the teeth with 
the most severe breakdown. ${ }^{7}$

The smoking habit and the IL-1 genotype of the patients seemed useful in predicting future risk for disease progress. Patients who smoked or were positive for IL-1 had a threefold increased risk of losing their teeth. Patients who were IL-1 positive and heavy smokers were nearly eight times more likely to lose teeth. ${ }^{4}$

\section{Success rates of periodontal therapy}

The results of most studies on the effectiveness of periodontal therapy are encouraging. Hirschfeld and Wasserman found that 7.1\% of the teeth were lost for periodontal reasons. Fifty per cent of the patients did not lose any teeth over a period of 22 years. ${ }^{1}$ McFall, in a duplicate study, had very similar results. ${ }^{8}$ Becker et al. showed comparable failure rates. When the teeth with an initial hopeless prognosis were excluded, the failure rate dropped by half $(2.94 \%) .{ }^{9}$

Even the tooth type has been shown to be a factor in the survival of the tooth. ${ }^{1,8,9}$ The tooth loss pattern was almost identical in Hirschfeld, Wasserman and McFall's studies. Maxillary molars were the teeth that are most likely to be lost, followed closely by mandibular molars. The maxillary and mandibular canines were the teeth most resistant to periodontal breakdown. ${ }^{1,8,9}$

McGuire concluded that it is easier to predict the prognosis for single-rooted teeth. ${ }^{2}$ Most studies seem to agree that anterior teeth respond better to periodontal treatment and are less likely to be lost due to periodontal reasons. None of the canines were lost in a well maintained population after 22 years of follow-up. ${ }^{1}$ Maxillary molars, on the other hand, had the worst prognosis. ${ }^{1,8}$ Ramfjord et al. found that the response of anterior teeth to periodontal treatment was marginally better than posterior teeth. The poorest results occurred for the maxillary bicuspids and molars, which may in part be related to furcation involvement ${ }^{10}$ and the time of the disease onset. In patients with mild periodontitis, the molar teeth were four times more likely to be affected than all other teeth combined. ${ }^{7}$ In patients with more advanced disease, $85 \%$ of the molar teeth presented with severe destruction. ${ }^{7}$ It follows that molars are "problem teeth' and the efficacy of different types of treatment must be explored.

\section{Success rates of surgical and non-surgical therapy on molars}

In the treatment of molar teeth there are various aspects that have to be investigated in order to evaluate the effectiveness of therapy. Teeth with and without furcation involvement have to be studied separately. Additionally, there are different therapeutic approaches for furcated molars. The treatment modalities include either preservation of the furca and strict maintenance or elimination of it by root amputation and hemisection.

Absence of furcation involvement The treatment outcome even in the absence of

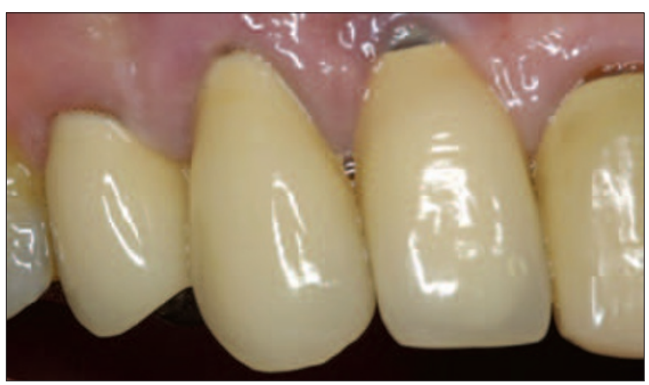

Fig. 5 Implant supported crown number 7 that presents with aesthetic problems. It is too long and metal display is evident in the cervical area.

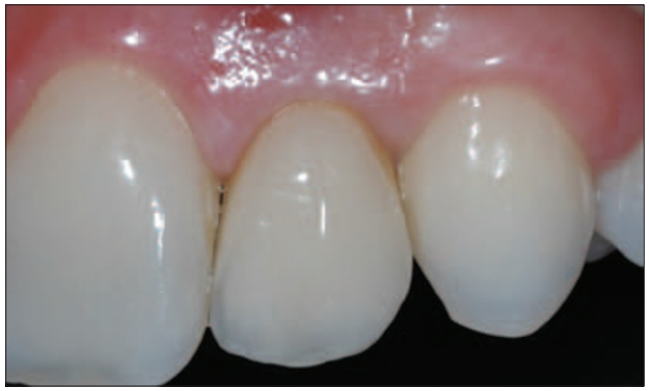

Fig. 6 Harmonious aesthetics can be achieved with implant restorations in region of tooth number 10 .

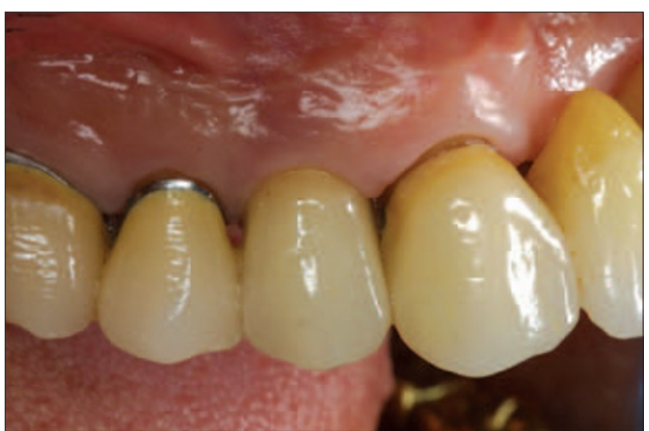

Fig. 7 Single crown on an implant replacing number 5 , seven years postoperatively.

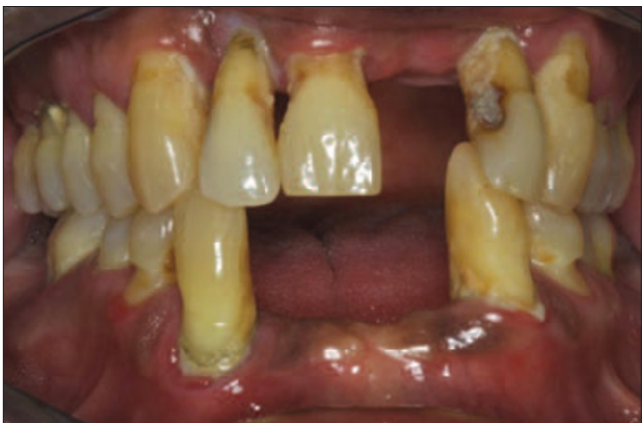

Fig. 8 Poor oral hygiene habits can be detrimental regardless of the level of treatment provided and this will be the overriding factor when treatment planning.

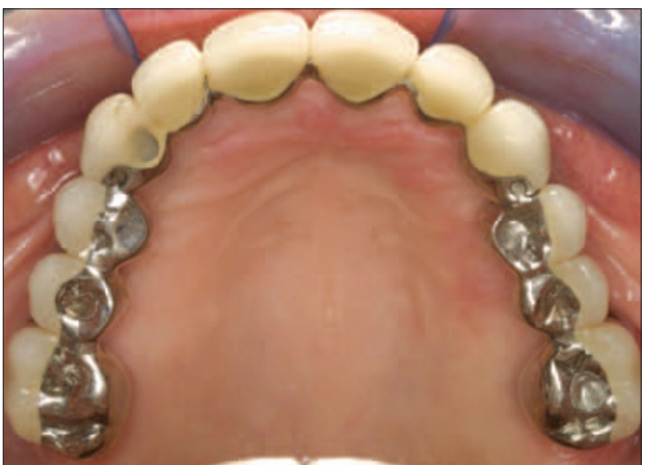

Fig. 9 Cross-arch splinting reconstruction due to periodontal disease.

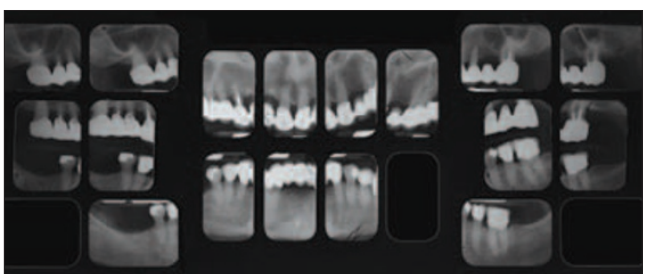

Fig. 10 Full mouth radiographs reveal caries on teeth number 7 and 12 and very small roots. 
Fig. 11 Implants were placed. The central incisors and first molars support a metal-reinforced fixed provisional restoration.

Fig. 12 CT scan cuts of right and left first molar areas showing insufficient bone volume to house implants.

Fig. 13 Tooth number 5 has a large diameter post, periapical radiolucency and needs a new crown.

Fig. 14 Implant-supported fixed partial denture on implants numbers 4 and 6.

Fig. 15 Failing endodontic therapy.
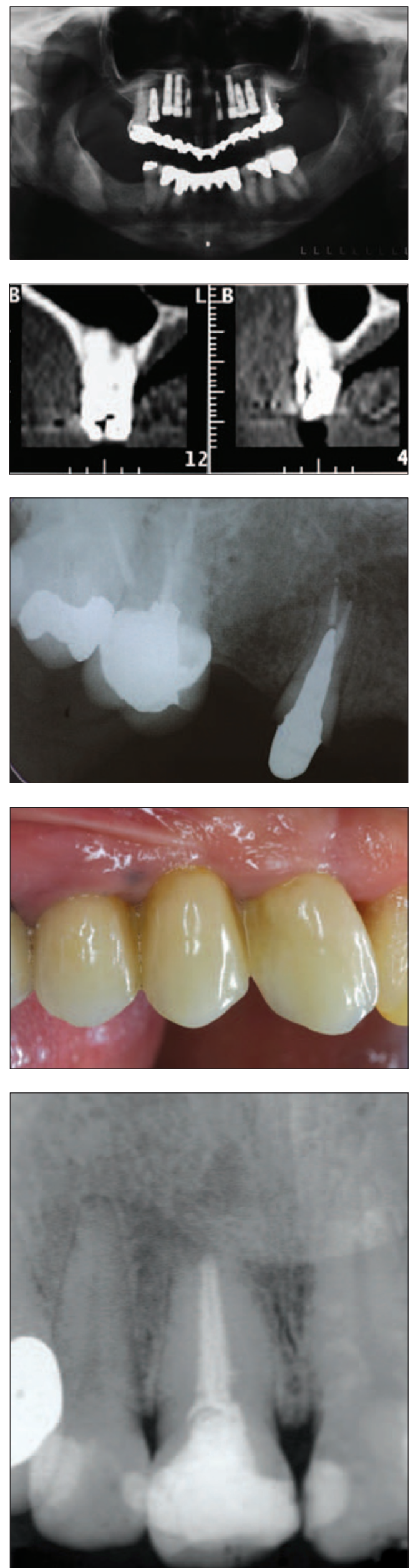

furcation involvement is problematic. However, the results are far more favourable compared to teeth with destruction in the furca. Sixty-four per cent of non-furcated teeth with a questionable prognosis were lost over a course of 19 years. ${ }^{8}$ When 323 molar teeth without furca invasion were followed for 6.5 years, $78 \%$ remained unchanged while the remaining $22 \%$ developed a furcation problem. ${ }^{9}$

Presence of furcation involvement without root resection or amputation Wang et al. concluded that in the presence of furcation involvement, teeth were twice as likely to be lost. ${ }^{11}$ Kalkwarf et al. observed that furcation sites tended to lose probing attachment levels regardless of the type of therapy provided. This may be a result of the inability to adequately instrument these areas during therapy. ${ }^{12}$

Without any root resection or hemisection procedure performed, Hirschfeld and Wasserman reported loss of nearly one third of the teeth originally diagnosed as having furcation invasion. ${ }^{1}$ McFall found that more than half of furca involved teeth were lost when followed from 15 to 29 years. ${ }^{8}$

Conversely, Ross and Thompson reported acceptable results with non-surgical intervention of maxillary molars with furcation involvement. After five to 24 years, $88 \%$ of the teeth were still functioning comfortably. However, the significance of these results is limited when one considers that an additional 11\% showed increased bone loss and that the diagnosis of all furcations was done solely on radiographs. ${ }^{13}$ Becker et al. published very similar results with the status of $86 \%$ of furcated molars remaining stable. Their conclusion was that teeth with moderate furcation involvement can be treated successfully and maintained effectively for prolonged periods. ${ }^{9}$

Furcation involved teeth receiving root resection or amputation Unfortunately, most studies of surgical intervention with root resection or hemisection do not present very promising results either. Langer et al. evaluated 100 patients receiving root resection therapy at least 10 years prior to the study. Thirty-eight per cent of these teeth failed, the majority occurring between the fifth and seventh year. Mandibular molars failed at a 2:1 ratio compared to maxillary molars. The latter failed primarily because of progressive periodontal disease, while mandibular molars succumbed most frequently to root fractures. ${ }^{14}$ Blomlöf et al. reported a very similar success rate of $68 \%$ at 10 years. Smokers seemed to have a threefold risk compared to non-smokers. ${ }^{15}$

A study which illustrated more promising results was conducted by Carnevale et al. They examined 488 hemisected or root resected teeth. The possible failure mode could have been periodontal, endodontic or restorative. The failure rate was 5.7\% and only 3.7\% of all the teeth had to be extracted. The highest cause of failure was dental caries and root fractures, but not periodontal disease. However, since this is a 
retrospective study, the number of furcated teeth that were initially extracted is not reported and thus conclusions about the efficacy of surgical treatment of the furcated teeth should be made with extreme caution. The authors explained the higher success rates compared to other studies by the fact that resection therapy is very technique-sensitive and proper case selection and restorative expertise are essential. ${ }^{16}$

\section{Importance of regular maintenance}

One aspect that all authors emphasise is the necessity for frequent recall appointments. The high success rates of Carnevale et al. are coupled with a three-month recall for 95\% of his patients. ${ }^{16}$ A frequency of three to four appointments per year is advocated for the periodontally involved patients. ${ }^{1,2,8,9}$

Achieving a proper maintenance program is not an easy task. Although the recall appointments were sent every three to four months, patients attended every 5.2 months. Additionally, by the seventh year after treatment, there was a $22.1 \%$ drop-out rate. ${ }^{9}$

Becker et al. reported that in a well-maintained population after 6.5 years, the annual tooth loss was 0.11 teeth per patient. ${ }^{9}$ The authors also examined another group of patients who did not return for recall for five years. Receiving treatment without maintenance had a negligible effect on reducing probing depths and $25 \%$ of shallow pockets became deeper. There was worsening of the furcation areas and statistically significant bone loss. Finally, the mean annual tooth loss doubled, reaching 0.22 teeth per patient. ${ }^{17}$

\section{Conclusion on periodontally involved teeth}

It is evident that, with the tools available today, accurate prognosis of periodontally involved teeth is unreliable. There are some guidelines that have prognostic value, but they should be used with caution. Survival rates of anterior teeth exceed that of posterior teeth. It follows that anterior teeth can be maintained with lower risk. However, in rare circumstances, even teeth with excellent periodontal status show rapid degradation (Figs 1 and 2). It is the multifactorial nature of the disease that makes prognosis and sometimes preservation of the teeth unpredictable.

\section{Success rates of non-surgical endodontic therapy}

When a tooth is fractured, grossly carious or traumatised, the choice for a patient may be either endodontic therapy or loss of the tooth. It is important for the patient and dental profession to be able to decide on a course of treatment through knowledge of potential success of various treatment modalities.

In a classical study on rats, Kakehashi et al. showed that in the absence of bacteria, complete healing of exposed dental pulps occurred. ${ }^{18}$ Sjögren et al. showed that when there was a periapical lesion present, endodontic success rates

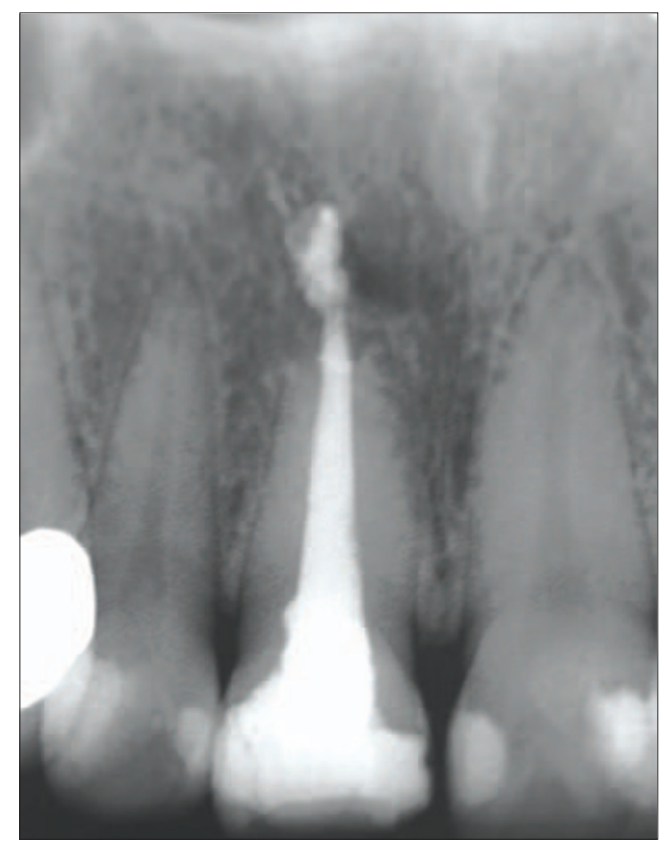

Fig. 16 The endodontist decided to perform apical surgery and retrograde root filling.

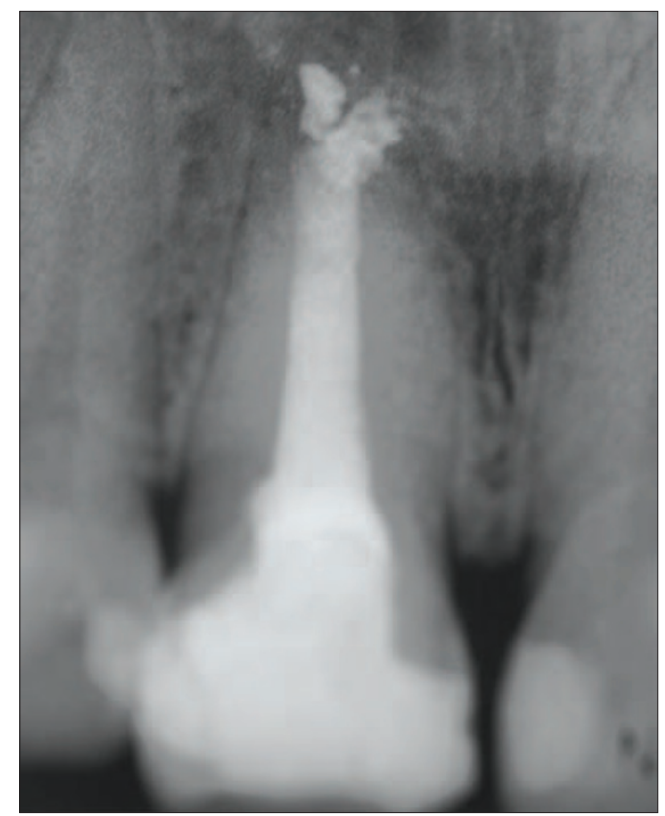

Fig. 17 Second apical surgery and retrograde root filling.

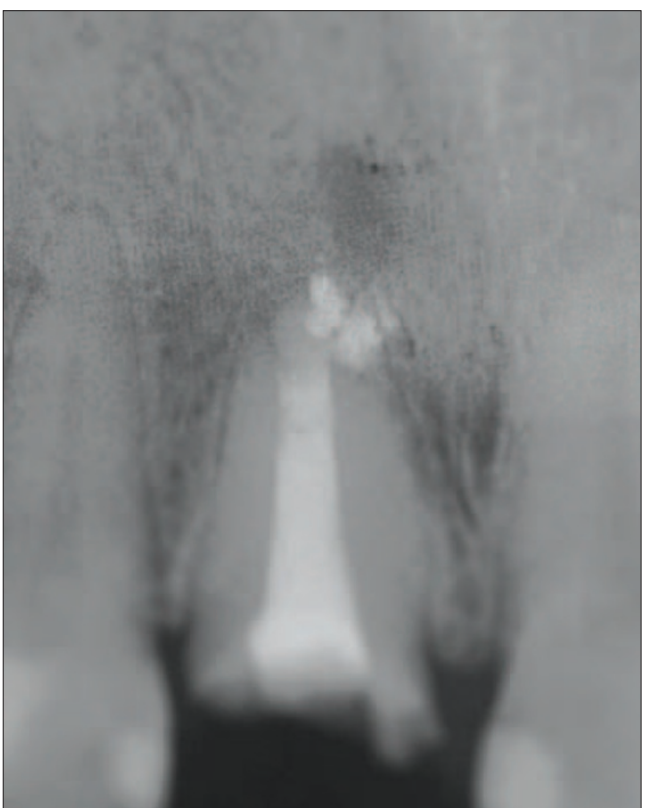

Fig. 18 Third apical surgery and retrograde root filling. Despite the endodontist's effort, the tooth was still symptomatic. 
Fig. 19 After the third surgery failed, the tooth was extracted. Remnants of the retrograde root filling can be observed on the radiograph.

Fig. 20 A fifth surgical procedure was necessary to remove the remnants.

Fig. 21 The root length was favourable and a narrow diameter for the dowel was intentionally maintained.

Fig. 22 This tooth received root cana therapy, crown lengthening, cast dowel and core and a new crown.

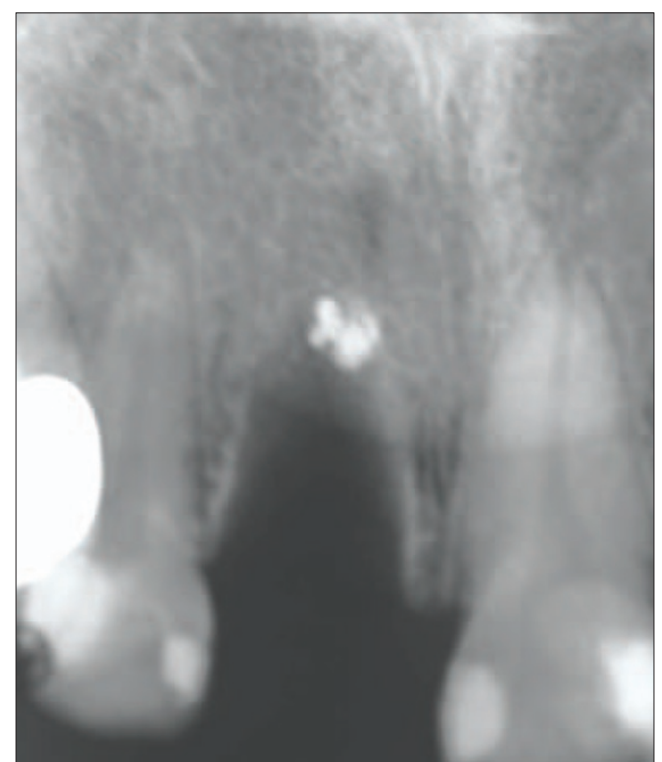

dropped by at least $10 \% .{ }^{19}$ They also showed that an initial negative culture resulted in a 94\% endodontic success rate, while an initial positive culture resulted in significantly reduced success rates $(68 \%) .{ }^{20}$ Fouad et al. demonstrated that '... in cases with preoperative periradicular lesions, a history of diabetes was associated with a significantly reduced outcome. ${ }^{21}$ The data suggest that patients who are diabetic and have an infected root canal may have a significantly reduced chance of healing from an endodontic infection.

Eriksen et al. showed that endodontic specialists achieve higher success rates when compared to general practitioners. They also showed that endodontic success rates varied between $54-94 \% .{ }^{22}$ In an investigation of nearly 2,500 teeth, Jonkinen et al. showed that success rates for endodontic therapy may be as low as 53\%.23 However, in this study the protocol for endodontic therapy differed from what is currently accepted as the norm. This may have had a negative influence on the success rates.

The real cause for confusion in survival studies seems to be the way in which the term 'success' is defined. If a study has strict criteria for success, the results are negatively affected. On the other hand, if the criteria are less strict, the success rates may be positively affected.

The reporting of success rates in endodontic literature can be confused by the definition of 'success/failure, the time period that the outcome was measured over, the type of endodontic procedure and the unit of measurement.

Much of the literature-cited success rates are dependent on resolution of the periodontal ligament space with radiographic findings alone and clinical symptoms are not considered. ${ }^{24-26}$ Furthermore, study periods are often not adequate to allow classification of teeth displaying a reduction in periapical radiolucency. Incomplete radiographic resolution and success rates from the longest period of follow-up are extrapolated to that of the mean period; measurement of success is based on roots rather than teeth or has not included teeth extracted. ${ }^{19,24}$

Friedman and Mor in 2004 defined success as root canal treatment that 'has healed' or 'is healing'. They also proposed a new classification: 'functional retention'. Functional retention is the sum of the healed and the healing sites. They also suggest that functional retention includes a tooth with a normal clinical presentation, where radiolucency is present or absent, newly emerged or persisting. ${ }^{27}$ In the opinion of the authors, although functional retention may result in higher apparent success rates, it may not lead to a predictable endodontic outcome. Functional retention is a loose criterion for assessment of endodontic success and may mislead the reader into believing that success rates are actually higher than they really are.

\section{Success rates of surgical endodontic therapy}

Friedman and Mor pooled data from selected studies and showed that the chance of success based on selected studies ranged from $37-85 \%$ 
with an average of 70\%. The chance of functionality for surgical endodontic procedures was $86-92 \% .27$ Again, functionality increases the numerical value for success rate of surgical endodontics. But one needs to decide whether a functional tooth will result in a predictable outcome.

\section{Restorability of endodontically treated teeth}

Another important issue is the restorability of endodontically treated teeth. Even if a tooth has been successfully treated with endodontics, one still needs to consider the restoration of the tooth. Goodacre et al. in a meta-analysis showed that in 12 studies with 2,784 teeth and a sixyear follow-up, $12 \%$ of teeth with dowels had complications. ${ }^{28}$ Many of these complications may lead to tooth loss (Figs 3-4). So, the practitioner needs to objectively assess the restorability of each endodontically treated tooth prior to commencement of treatment. The predictability of the treatment provided will be of benefit both to the patient and dentist.

\section{Success rates of dental implants}

In an attempt to objectively quantify success with regards to dental implants and their restorations, many criteria have been defined. The implants should have a minimum of one year of loading, as most implant failures are detected in the first year of service. ${ }^{29,30}$ Implant failures should also be defined. It is suggested that if an implant can not be used as support for prosthetic reconstruction, it should be labelled a 'sleeping implant'. These are labelled surviving implants at best, as they are not usable. ${ }^{31}$ Lindh et al. suggested that these should really be classed as failures. If sleeping implants are osseointegrated, they should be regarded as 'functional failures' because they are unrestorable. ${ }^{31}$

Smith and Zarb have suggested that the aesthetic aspect of the implant position should also be incorporated as factors for a successful result. ${ }^{32}$ In another meta-analysis Goodacre et al. showed that 47 out of 493 crowns/prostheses produced aesthetic problems. They found that aesthetic failures had a mean of 10\% (Figs 5 and 6).

Gibbard and Zarb stated that 'Long-term success for multiple splinted implants cannot be extrapolated to single implants.33 In a meta-analysis of 66 studies over 10 years, Lindh et al. included 2,686 dental implants, and evaluated 570 single crowns and 2,116 implant fixed partial dentures in partially edentulous jaws. '...Although the cumulative survival rate will decrease if 'sleeping implants' are considered as failed, the maximum difference is only $3.7 \%$...' Implant survival under load after six to seven years was $93.6 \%$ for fixed partial dentures and $97.5 \%$ for single crowns ${ }^{27}$ (Fig. 7). The data from the Lindh et $a .^{31}$ study suggest that implants and their restorations work extremely predictably for single teeth or fixed partial dentures. It also shows that even with strict inclusion criteria these restorations have excellent success rates.

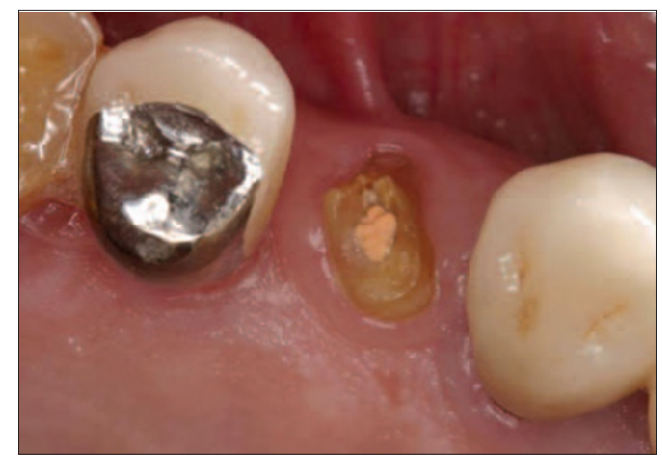

Fig. 23 This tooth requires endodontic re-treatment, crown lengthening, dowel and core and a crown. It was decided to extract the tooth as the patient was a bruxer and the root had unfavourable anatomy.

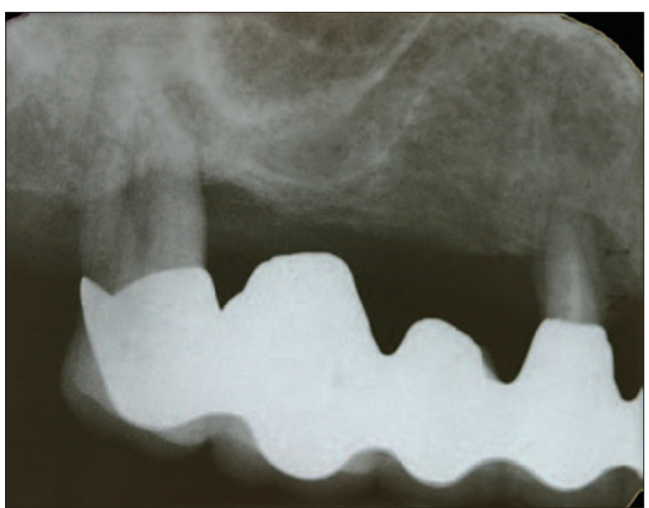

Fig. 24 Advanced periodontal disease. The treatment of choice was extraction and implant placement.

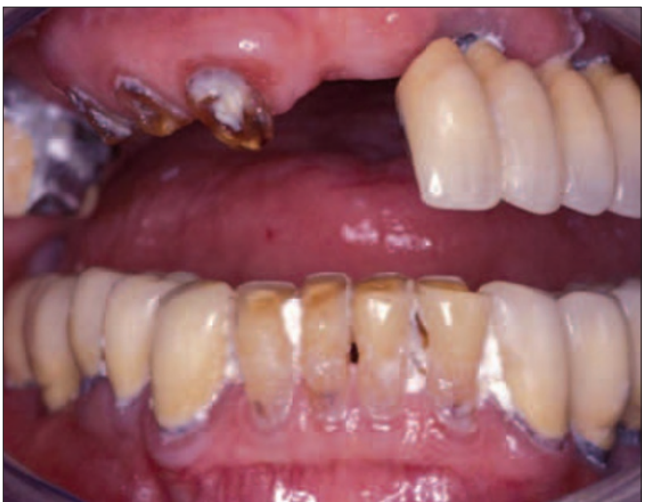

Fig. 25 This patient has implant supported restorations in the maxillary left and mandibular posterior quadrants. Note failing natural dentition.

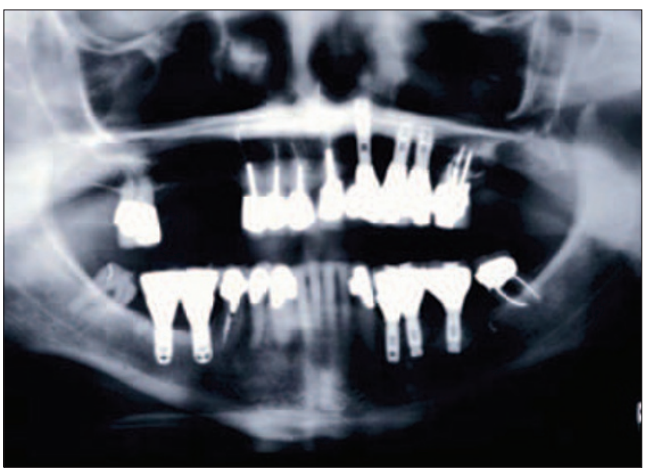

Fig. 26 Panoramic radiograph of patient in Figure 25.

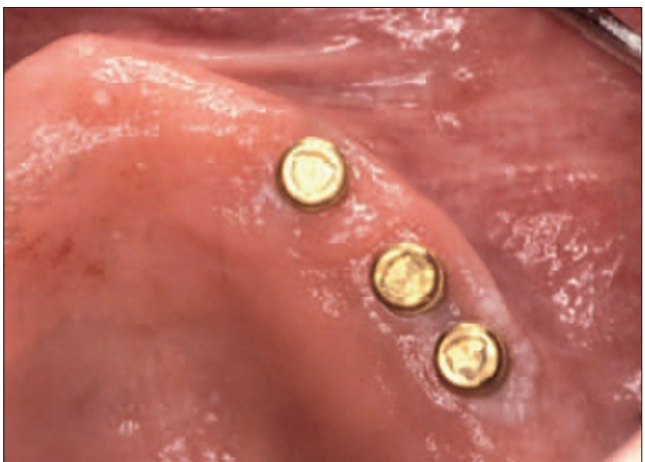

Fig. 27 Same patient as in Figure 25. Natural dentition in maxilla has failed. Due to patient health additional implants could not be placed. A maxillary denture was fabricated which was implant retained and mucosa supported. 
Fig. 28 Maxillary denture with Vitallium Palate.

Fig. 29 Panoramic radiograph showing single implant placement in mandible. Remaining mandibular teeth are failing.

Fig. 30 Mandibular teeth have failed additional implants have been placed.

Fig. 31 Same patient as in Figures 29-30. There are sufficient implants placed for a fully implant supported restoration
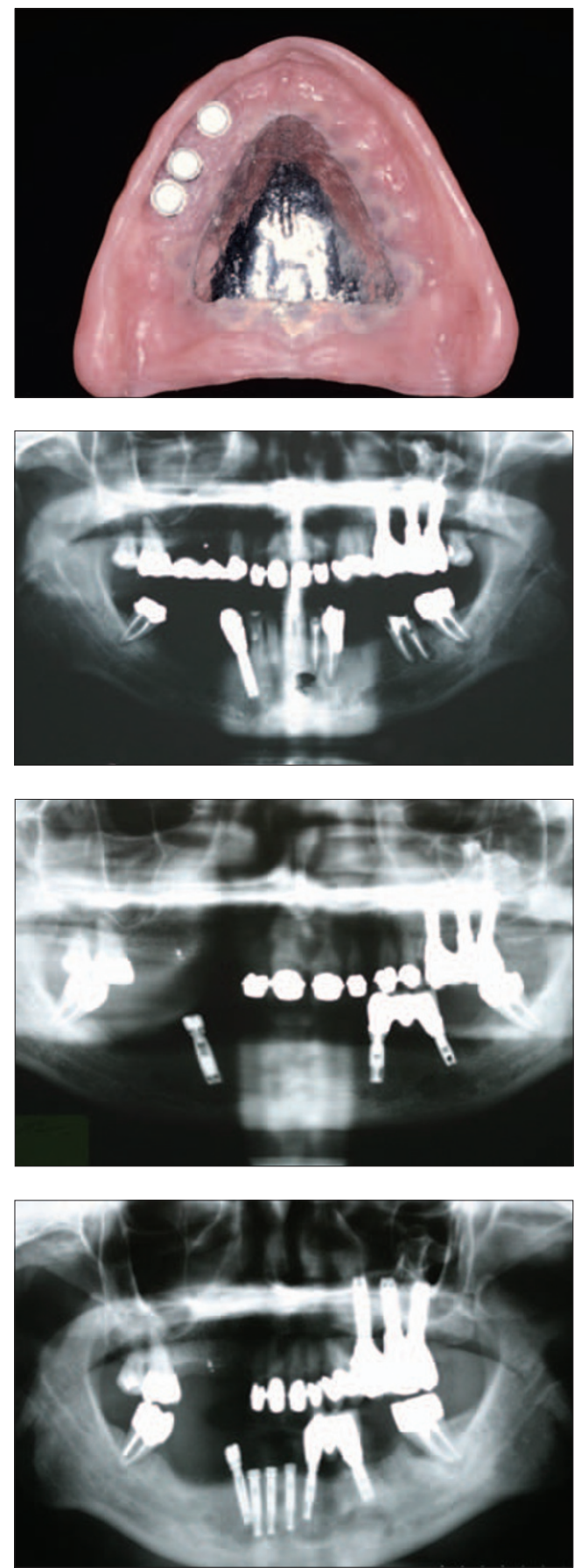

\section{DISCUSSION}

Unfortunately, there are no rules or formulae in dentistry that provide straightforward answers. The practitioner needs to use the knowledge from the literature along with common sense to derive a treatment plan. The picture is further complicated by a multitude of local, systemic and even psychological factors. The patient's medical conditions, the general condition of the oral environment and certainly the patient's motivation towards the treatment will influence the overall success (Fig. 8). Thus, the actual longevity of a specific treatment modality cannot be applied to all patients indiscriminately.

Considering all these parameters, the clinician is often faced with a dilemma when deciding whether or not to extract a tooth with a poor prognosis. Traditional wisdom was based upon the concept of trying to save the tooth by all means necessary. However, with the inception of dental implants a completely new avenue has been opened in the treatment planning process. This has created a new topic for debate within the profession. There appears to be two schools of thought. One school is advocating the traditional approach, while the other has adopted a more aggressive approach with treatment planning and prefers to extract and replace a compromised tooth with a dental implant and restoration.

It is imperative to understand that each therapeutic modality has an inherent biological cost. Therefore, a risk analysis should be initiated prior to any definitive decisions. In the authors' opinion a very stringent approach is required during this analysis. A treatment with a poor risk-to-benefit ratio has a greater probability of biological consequences. In treatment planning, DeVan's statement should always be a cornerstone in the dentist's mind: '...our goal should be the preservation of what remains rather than the meticulous restoration of what is missing. 34

Nyman and Lindhe have shown excellent results with the prosthetic rehabilitation of patients with advanced periodontal disease with very few prosthetic complications. ${ }^{35}$ Figure 9 illustrates a patient that received full mouth reconstruction in the maxilla due to moderate periodontal disease. After 25 years the osseous support did not show significant changes with regular periodontal maintenance (Fig. 10). Nevertheless, the patient's medical status changed and the salivary flow decreased significantly. The result was caries development on two abutment teeth (Fig. 10). Considering the medical history along with the success rates of different treatment modalities, it was decided to extract most of the maxillary teeth and place implants (Fig. 11). Under no circumstances can the previous periodontal-prosthetic rehabilitation be considered a failure after 25 years of survival. An implant-supported restoration was chosen over a tooth-borne cross-arch splint. This decision was based on the obvious risk associated with joining numerous teeth in a medically compromised patient.

The strategic value of the tooth must also be assessed. In the patient shown in Figure 11, the most distal molars were maintained. Extractions would have resulted in sinus lifting procedures, which the patient wished to avoid (Fig. 12). Although the teeth had a guarded prognosis, their value as two additional occluding units contradicted their removal. Figure 13 shows tooth number 5 that has a large cast dowel and needs endodontic re-treatment and a new crown. Implants are planned for the mesial and distal edentulous sites, while the existing teeth on the mesial and distalsites do not need restorations or replacement of restorations. Due to the risk involved and the low strategic value of the tooth, a three-unit implant-supported fixed partial denture was fabricated (Fig. 14). It can 
be deducted that teeth with higher strategic value will be amenable to more extensive procedures than teeth in less important positions in the arch.

\section{Clinical recommendations and conclusions}

Implant placement and restoration is not a technically-demanding procedure. ${ }^{36,37}$ From the results available today, which are based on follow up studies, it seems that tooth replacement with dental implants is more predictable than surgical periodontal and endodontic techniques (Figs 15-20). This however should not automatically preclude these therapeutic modalities and lead to extraction of the affected teeth. It does justify, though, a relatively more aggressive approach, especially in younger patients where a significantly long-term prognosis is required.

One needs to decide on the most predictable strategy for restoring a severely broken-down tooth. This may involve the combination of endodontic, periodontal and restorative procedures, in order to save a tooth (Figs 21-22). On the other hand, what has been considered successful prior to the inception of dental implants might not be acceptable today. If the tooth has minimal coronal tooth tissue remaining with unfavourable root structure or if multiple procedures need to be performed, one is justified in extracting the tooth in favour of a dental implant (Fig. 23). Multiple procedures, even if independently low-risk, significantly increase the risk of failure. On the other hand, removal of all teeth that do not receive a good prognosis is extremely aggressive and contraindicated.

Heroic attempts to maintain teeth with poor prognosis should cease. Such attempts increase the risk for failure, as well as the cost for the patient in the long run. They may also jeopardise future treatment outcomes. For example, as periodontal destruction progresses, the risk of insufficient bone volume for implant placement increases. Historically the teeth shown in Figure 24 would be maintained until they would exfoliate from the patient's mouth. A more aggressive approach, nowadays, will save the patient from the high morbidity and lower predictability of bone grafting procedures. The authors believe that the interpretation of the '...preservation of what remains ${ }^{34}$ should be extended to the precious osseous structure of the ridges.

Another aspect to consider is that dental caries and periodontal disease do not affect implants as there is no calcified tissue and no periodontium. Implants seem to withstand the ravages of the oral cavity better than teeth do (Figs 25-28). Implant restorations can also be planned for retrievability (Figs 29-31). As teeth are lost, restorations can be modified and connected to additional implants. Long term treatment planning to allow for retrievability becomes important; once implants integrate they can be almost considered life-time anchorage units. Clinicians today must become well acquainted with the use of implants in restorative dentistry, implants should not be "the last resort' and only used when all conventional therapies have failed. There is sufficient documentation today to indicate the use of implants as the first choice of treatment in many situations. With this knowledge the fine line of whether to maintain a tooth or to replace it with an implant supported restoration must be made. With knowledge of the literature regarding success rates of therapies and survival rates of restorations, the clinician has the responsibility to help patients make the choice. A risk benefit and a cost benefit analysis should be carried out each time.

The authors would like to thank the Journal of the California Dental Association for permission to reprint parts of this article which were initially published in the JCDA April 2005 issue.

1. Hirschfeld A, Wasserman B. A long-term survey of tooth loss in 600 treated periodontal patients. J Periodonto/ 1978; 49: 225-237.

2. McGuire M K. Prognosis versus actual outcome: a longterm survey of 100 treated periodontal patients under maintenance care. J Periodontol 1991; 62: 51-58.

3. McGuire M K, Nunn M E. Prognosis versus actual outcome. II. The effectiveness of clinical parameters in developing an accurate prognosis. J Periodonto/ 1996; 67: 658-665.

4. McGuire M K, Nunn M E. Prognosis versus actual outcome. IV. The effectiveness of clinical parameters and IL-1 genotype in accurately predicting prognoses and tooth survival. J Periodontol 1999; 70: 49-56.

5. McGuire M K, Nunn M E. Prognosis versus actual outcome. III. The effectiveness of clinical parameters in accurately predicting tooth survival. J Periodontol 1996; 67: 66-74.

6. Lang N P, Joss A, Orsanic T, Gusberti F A, Siegrist B E. Bleeding on probing. A predictor for the progression of periodontal disease? J Clin Periodonto/ 1986; 13: 590-596.

7. Wasserman B H, Thompson R H Jr, Geiger A M. Relationship of occlusion and periodontal disease. II. Periodontal status of the study population. J Periodonto/ $1971 ; 42: 371-378$.

8. McFall W T Jr. Tooth loss in 100 treated patients with periodontal disease. A long-term study. J Periodontol 1982; 53: 539-549.

9. Becker W, Berg L, Becker B E. The long term evaluation of periodontal treatment and maintenance in 95 patients. Int J Periodontics Restorative Dent 1984; 4: 54-71.

10. Ramfjord S P, Knowles J W, Morrison E C, Burgett F G, Nissle $R$ R. Results of periodontal therapy related to tooth type. J Periodontol 1980; 51: 270-273.

11. Wang $H-L$, Burgett F G, Shyr Y, Ramfjord S. The influence of molar furcation involvement and mobility on future clinical periodontal attachment loss. J Periodonto/ 1994; 65: 25-29.

12. Kalkwarf K L, Kaldahl W B, Patil K D. Evaluation of furcation region response to periodontal therapy. J Periodonto/ 1988 ; 59: 794-804.

13. Ross I F, Thompson R H. Long term study of root retention in the treatment of maxillary molars with furcation involvement. J Periodonto/ 1978; 49: 238-273.

14. Langer B, Stein SD, Wagenberg B. An evaluation of root resections. A ten-year study. J Periodonto/ 1981; 52: 719-722.

15. Blomlöf L, Jansson L, Appelgren R, Ehnevid H, Lindskog S. Prognosis and mortality of root-resected molars. Int J Periodontics Restorative Dent 1997; 17: 190-201.

16. Carnevale G, Di Febo G, Tonelli M P, Marin C, Fuzzi M. A retrospective analysis of the periodontal-prosthetic treatment of molars with interradicular lesions. Int J Periodontics Restorative Dent 1991; 11: 189-205.

17. Becker W, Becker B E, Berg L E. Periodontal treatment without maintenance. A retrospective study in 44 patients. J Periodontol 1984; 55: 505-509.

18. Kakehashi S, Stanley H R, Fitzgerald R J. The effects of surgical exposures of dental pulps in germfree and conventional laboratory rats. J South Calif Dent Assoc 1966; 34: 449-451.

19. Sjogren U, Hagglund B, Sundqvist G, Wing K. Factors affecting the long-term results of endodontic treatment. JEndod 1990; 16: 498-504. 
20. Sjogren U, Figdor D, Persson S, Sundqvist G. Influence of infection at the time of root filling on the outcome of endodontic treatment of teeth with apical periodontitis. Int Endod J 1997; 30: 297-306.

21. Fouad A F. Diabetes mellitus as a modulating factor of endodontic infections. J Dent Educ 2003; 67: 459-467.

22. Eriksen H M. Endodontology - epidemiologic considerations. Endod Dent Traumatol 1991; 7: 189-195.

23. Jokinen M A, Kotilainen R, Poikkeus P, Poikkeus R, Sarkki L. Clinical and radiographic study of pulpectomy and root canal therapy. Scand J Dent Res 1978; 86: 366-373.

24. Morse D, Esposito J V, Pike C, Furst M L. A radiographic evaluation of the periapical status of teeth treated by the gutta-percha-eucapercha method: a one-year follow-up study of 458 root canals - Part III. Oral Surg 1983; 56: 190-197.

25. Seltzer S, Bender I B. Factors affecting successful repair after root canal therapy. J Am Dent Assoc 1963; 67: 651-662.

26. Heling I, Bialla-Shenkman S, Turetzky A, Horwitz J, Sela J. The outcome of teeth with periapical periodontitis treated with nonsurgical endodontic treatment: a computerized morphometric study. Quintessence Int 2001; 32: 397-400.

27. Friedman $\mathrm{S}$, Mor $\mathrm{C}$. The success of endodontic therapy - healing and functionality. J Calif Dent Assoc 2004; 32: 493-503.

28. Goodacre C J, Bernal G, Rungcharassaeng K, Kan J Y. Clinical complications in fixed prosthodontics. J Prosthet Dent 2003; 90: $31-41$.
29. Goodacre C J, Kan J Y, Rungcharassaeng K. Clinical complications of osseointegrated implants. J Prosthet Dent 1999; 81: 537-552

30. Goodacre C J, Bernal G, Rungcharassaeng K, Kan J Y. Clinical complications with implants and implant prostheses. Prosthet Dent 2003; 90: 121-132.

31. Lindh T, Gunne J, Tillberg A, Molin M. A meta-analysis of implants in partial edentulism. Clin Oral Implants Res 1998; 9: 80-90.

32. Smith D E, Zarb G A Criteria for success of osseointegrated endosseous implants. J Prosthet Dent 1989; 62: 567-570.

33. Gibbard L L, Zarb G. A 5-year prospective study of implantsupported single-tooth replacements. J Can Dent Assoc 2002; 68: 110-116

34. DeVan M M. Biological demands of complete dentures. J Am Dent Assoc 1952; 45: 524-527.

35. Nyman S, Lindhe J. A longitudinal study of combined periodontal and prosthetic treatment of patients with advanced periodontal disease. J Periodonto/ 1979; 50: 163-169.

36. Bell FA, Cavazos E J et al. Four-year experience with the placement, restoration, and maintenance of dental implants by dental students. Int J Oral Maxillofac Implants 1994; 9: 725-731.

37. Cummings J, Arbree N S. Prosthodontic treatment of patients receiving implants by predoctoral students: fiveyear follow-up with the IMZ system. J Prosthet Dent 1995; 74: 56-59. 